\section{Intracellularly grown gold nanoparticles as potential surface-enhanced Raman scattering probes}

\author{
Ali Shamsaie, Magdalena Jonczyk, Jennie Sturgis, \\ J. Paul Robinson, and Joseph Irudayaraj* \\ Purdue University, Department of Agricultural and Biological \\ Engineering and Bindley Biosciences Center, 225 South \\ University Street, West Lafayette, Indiana 47907
}

\begin{abstract}
Gold nanoparticles grown within the intracellular confines of living cells are introduced as potential surface-enhanced Raman scattering (SERS) substrates for confocal Raman spectrometry. Electron microscopy and a silver-enhanced reflectance laser scanning confocal microscopic approach were used to visualize the size, shape, and distribution of intracellularly grown gold nanoparticles (IGAuN) as small as $1 \mathrm{~nm}$. Passive uptake as the conventional approach for delivering nanoparticles inside cells faces the insurmountable challenge of escaping the endosomal/lysosomal pathway. In contrast, IGAuN provides an unprecedented advantage of providing access to cytoplasm and nucleus. ๑ 2007 Society of Photo-Optical Instrumentation Engineers. [DOI: 10.1117/1.2717549]
\end{abstract}

Keywords: surface-enhanced Raman scattering; confocal Raman microscopy; confocal reflectance microscopy; gold formation; silver enhancement; intracellular growth.

Paper 06298LR received Oct. 22, 2006; revised manuscript received Jan. 2, 2007; accepted for publication Jan. 8, 2007; published online Apr. 9, 2007.

High selectivity and ultra-high sensitivity offered by surface-enhanced Raman scattering (SERS) have significant potential in biological research. Enhanced viability and minimal collection times, when combined with improved resolution of modern confocal Raman microscopes, makes SERS a unique platform for Raman studies of living human cells. Metallic nanoparticles (mainly gold and silver) have been used almost exclusively as SERS substrates to monitor cellular processes and events. Examples of such studies include: enzymedrug interaction, ${ }^{1}$ probing intracellular $\mathrm{pH},{ }^{2}$ detecting intracellular constituents, ${ }^{3}$ and tracking exogenous molecules inside the cell. ${ }^{4}$ In these studies, "passive uptake" of these nanoparticles by cells has been the predominant approach to deliver nanoparticles inside the cells. However, this approach has major limitations: first, lack of control over aggregation and/or distribution of nanoparticles inside the cell, and second, very poor (almost none) translocation efficiency of nanoparticles to the cytoplasm or nucleus. It has been shown that nanoparticles as small as $10 \mathrm{~nm}$ after incubation and passive uptake are entrapped inside vesicles or membrane-bound organelles (most likely the lysosomes or endosomes) inside cells and could not find their way into the cytoplasm or nucleus. ${ }^{5}$ To achieve suitable SERS signals, the size of nanoparticles should be in the range between 20 to $60 \mathrm{~nm} .{ }^{6,7}$ Nanoparticles

*Tel: 765-494-0388; E-mail: josephi@purdue.edu
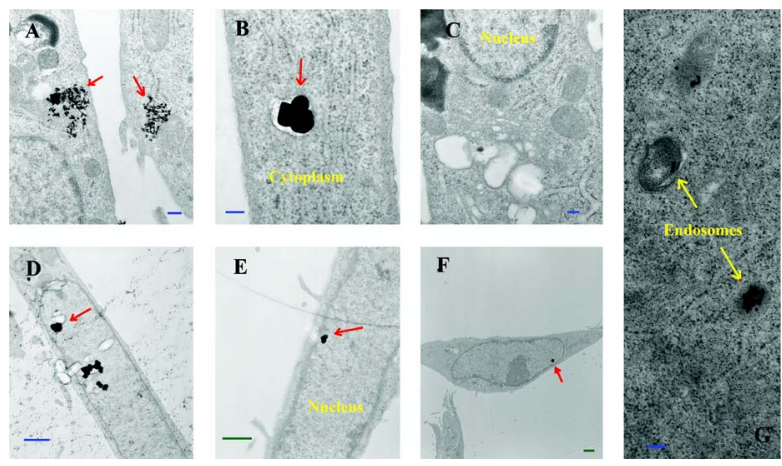

Fig. 1 TEM images showing IGAuNs of varing size and shapes inside (a) and (b) the cytoplasm and nucleus (d), (e), and (f). IGAuNs of smaller size $(<5 \mathrm{~nm})$ are spread throughout the nucleus, and (c) cytoplasm are not clearly visible in TEM images. In contrast to IGAuNs that are free within the cytoplasm, (g) 20-nm spherical gold nanoparticles are entrapped in the endosomal compartments after passive uptake. Magnifications: (a) $\times 27500$, (b) $\times 52000$, (c) $\times 27500$, (d) $\times 11000$, (e) $\times 12000$, (f) $\times 3800$, and $(\mathrm{g}) \times 27500$. Blue and green scale bars denote $1 \mathrm{~nm}$ and $1 \mu \mathrm{m}$ respectively (color online only).

of such sizes cannot escape the inevitable endosomal/ lysosomal pathway of the cell, and even if they did, they are too big to pass through the pores of the nuclear membrane $(<30 \mathrm{~nm})$.

Growing nanoparticles inside cells might be an alternative and unique approach to provide the enhancing element (SERS) and access to the innermost confines of the cellular microcosm, which are otherwise not possible by passive uptake. It has been known for a long time that certain microorganisms have the capacity to reduce metallic ions into elemental metals. Nanoparticles of different shapes, sizes, and compositions can be synthesized using different micoorganisms (bacteria, fungus, yeast, actinomycetes). ${ }^{8,9}$ The exact mechanism of the reduction process has not been elucidated yet. This reducing capacity has been shown by Anshup et al. for human cell lines. ${ }^{10}$ In this work, we provide a venue for using intracellularly grown Au nanoparticles (IGAuNs) as a SERS probe to obtain SERS spectra of the cytoplasm and/or the nucleus of a single cell.

MCF10 epithelial cells were used as model mammalian cells to investigate the active approach. 1-mM $\mathrm{HAuCl}_{4}$ solution in phosphate buffer saline $(\mathrm{pH}$ 7.4) was used for this purpose. To test for the formation of gold nanoparticles, cells were grown to confluency and the growth media was removed (to eliminate phenol red in the media, which is an interferent ${ }^{10}$ ) and replaced with phosphate-buffered saline (PBS) and incubated with $1-\mathrm{mM} \mathrm{HAuCl}_{4}$ in culture wells. The appearance of a pink color after $36 \mathrm{~h}$ indicated the formation of gold nanoparticles. No color change was observed in the negative controls (cells incubated with PBS without $\mathrm{HAuCl}_{4}$ ). Solutions of $\mathrm{HAuCl}_{4}$ in PBS are stable for months and hence the possibility of autoreduction is ruled out. Formation of elemental gold in PBS was further confirmed by the plasmon resonance band in the visible region $(\sim 530 \mathrm{~nm})$ of the absorption spectrum.

TEM images of thin cross sections of cells incubated with chloroaurate ion solution after $24 \mathrm{~h}$ clearly reveal the forma-

1083-3668/2007/12(2)/020502/3/\$25.00 @ 2007 SPIE 

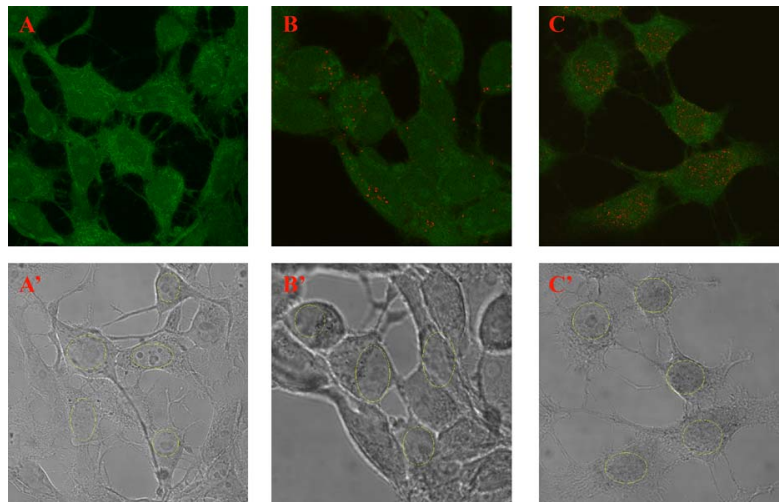

Fig. 2 Silver-enhanced laser scanning confocal images of intracellular gold particles. Reflectance versus transmission images of $(A)$ and $\left(A^{\prime}\right)$ negative control cells, $(B)$ and $\left(B^{\prime}\right)$ positive control cells with no nuclear traslocation, and $(C)$ and $\left(C^{\prime}\right)$ cells with IGAuNs inside nucleus and cytoplasm. All three groups, including the negative control, were subject to the same silver-enhancement protocol. Yellow ovals indicate the nucleus boundaries (color online only).

tion of gold nanoparticles of irregular shapes and varying sizes scattered throughout the cytoplasm [Figs. 1(a) and 1(b)]. Some of these IGAuNs form aggregates that are dramatically large, approaching the micrometer size range in a way that is visible even under a regular light microscope. The presence of smaller size IGAuNs (less than $5 \mathrm{~nm}$ ) within the nucleus and cytoplasm was suspected from the TEM images [Fig. 1(c)]. Since this size range is very close to the resolution limit of
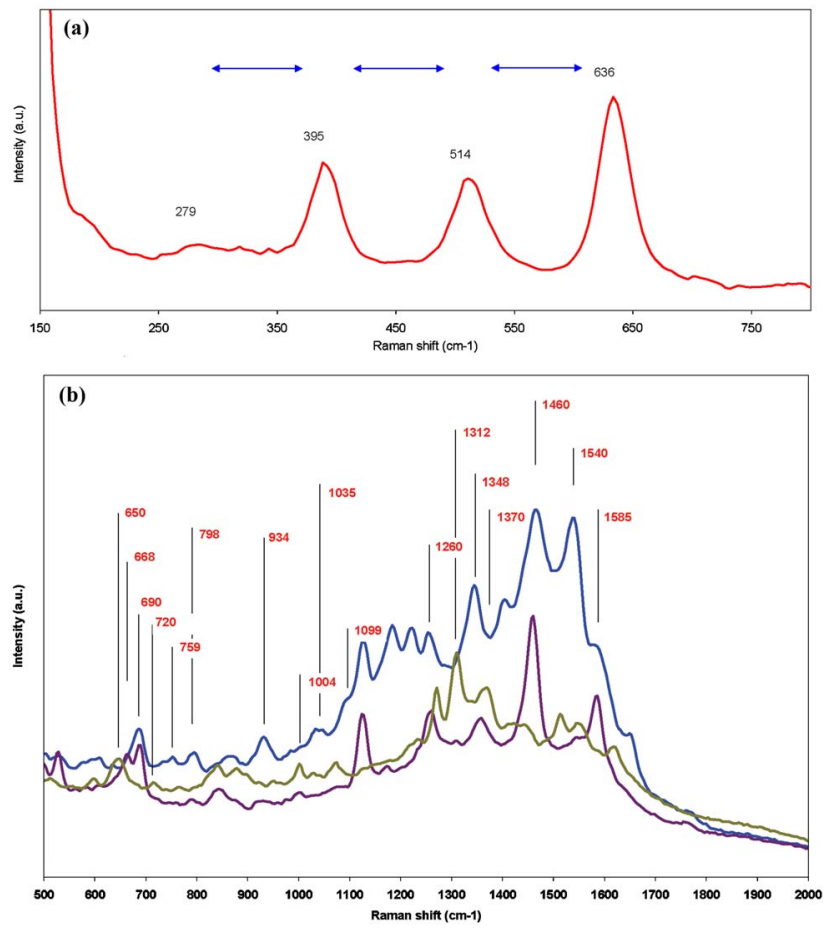

Fig. 3 (a) Low wavenumber lattice vibrations of IGAuNs depict their crystalline nature. (b) IGAuN-induced SERS spectra. Raman bands are evidence of sensing cytoplasmic or nuclear matrix (protein, DNA/ $\mathrm{RNA}$ ). Exposure time was $10 \mathrm{sec}$ and laser $(785 \mathrm{~nm})$ power was $10 \mathrm{~mW}$.
TEM, it is very difficult to distinguish IGAuNs from artifacts or ribosomes. Using reflectance laser scanning confocal microscopy (LSCM) combined with silver enhancement (also called autometallography), it is possible to visualize and track gold nanoparticles as small as $1 \mathrm{~nm}$. Gold nanoparticles can selectively nucleate the deposition of dense silver particles, which lead to their enlargement and enhanced visualization using appropriate confocal techniques. ${ }^{11,12}$ LSCM imaging from three groups of samples including negative control, positive control (cells incubated with 50-nm gold nanoparticles), and test (cells with IGAuNs) are presented in Fig. 2. The LI Silver kit (Nanoprobes, Incorporated, Yaphank, New York) was used for silver enhancement. As opposed to positive control, which shows little or no sign of gold nanoparticles inside the nucleus, cells with IGAuNs show a large amount of gold nanoparticles deposited inside the nucleus as well as the cytoplasm. While the true mechanism of intranucleus formation of gold nanoprticles is not clearly understood, previous studies have implicated the role of membrane sugars and enzymes in the reduction process. ${ }^{13}$ Hence one can speculate that a portion of smaller IGAuNs produced in the nuclear membrane may have diffused or translocated to the nuclear matrix. A similar mechanism has been proposed in the study of cytoplasmic IGAuNs during fungus mediated synthesis of silver nanoparticles. ${ }^{14}$ Apart from these small size IGAuN inside the nucleus, we have also noted the formation of relatively very large size spherical nanoparticles inside the nuclues [Figs. $1(\mathrm{~d})-1(\mathrm{f})]$. To our knowledge, this is the first report on the formation of such large nanoparticles (up to $300 \mathrm{~nm}$ ) inside the nucleus of a single cell.

SERS spectra were collected using a confocal Raman microscope fitted with a $60 \times$ water immersion objective and a 785-nm near-infrared (NIR) laser. Spatial and spectral resolutions were about $1 \mu \mathrm{m}$ and $10 \mathrm{~cm}^{-1}$, respectively. Laser power was set to $10 \mathrm{~mW}$ and the laser spot size was estimated to be lower than $2 \mu \mathrm{m}$. Spectra were collected both in a random fashion and also specifically from the dark spots believed to be IGAuN aggregates. The majority of Raman spectra collected from the IGAuN aggregates show a non-SERS pattern depicted in Fig. 3(a), which includes four distinct bands around 279, 395, 514, and $636 \mathrm{~cm}^{-1}$. Careful observation reveals that these bands are equally spaced by almost the same value [Fig. 3(a)] which is indicative of the resonance effect

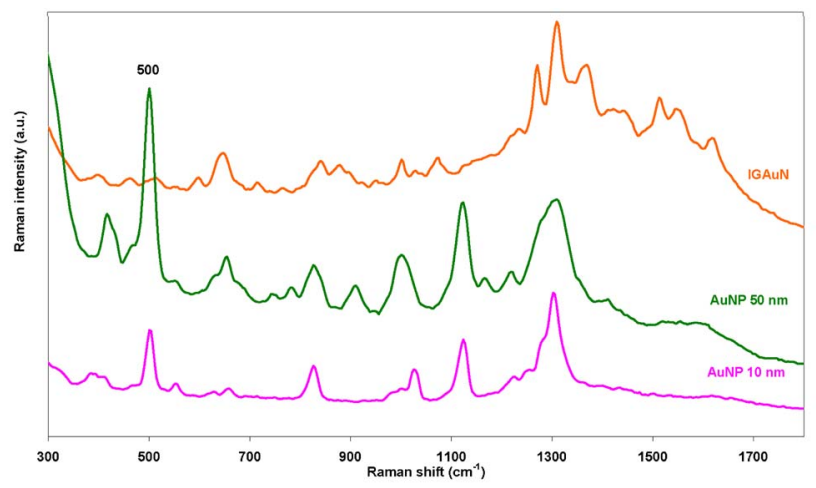

Fig. 4 Comparison between IGAuN-induced and passively uptaken gold nanoparticle (AuNP) (10 and $50 \mathrm{~nm}$ ) induced SERS. Exposure time was $1 \mathrm{sec}$ and laser $(785 \mathrm{~nm})$ power was $10 \mathrm{~mW}$. 
arising from the lattice vibrations of crystals. Such characteristic vibrations could not be observed in the Raman measurements of samples incubated with spherical gold nanoparticles. SERS spectra collected from IGAuNs, on the other hand, look quite different and highly resolved. Figure 3(b) shows three sample Raman spectra in the fingerprint region, collected from single living cells with IGAuNs. Considering the physical location of IGAuNs, we hypothesize that IGAuNs could potentially sense the nuclear and/or cytoplasmic materials. Many of the bands in these spectra can be ascribed to DNA and/or proteins. For example: $650 \mathrm{~cm}^{-1}$ (guanine), $668 \mathrm{~cm}^{-1}$ (DNA), $720 \mathrm{~cm}^{-1}$ (adenine), $759 \mathrm{~cm}^{-1}$ (tryptophan), $798 \mathrm{~cm}^{-1}$ (DNA/RNA), $934 \mathrm{~cm}^{-1}$ (protein backbone), $1004 \mathrm{~cm}^{-1}$ (phenylalanine), $1035 \mathrm{~cm}^{-1}$ (protein), $1099 \mathrm{~cm}^{-1}$ (DNA backbone), $1260 \mathrm{~cm}^{-1}$ (amide III), $1312 \mathrm{~cm}^{-1}$ (protein, DNA/RNA), $1348 \mathrm{~cm}^{-1}$ (protein, DNA/ RNA), $1370 \mathrm{~cm}^{-1}$ (protein, DNA/RNA), $1460 \mathrm{~cm}^{-1}$ (protein, DNA/RNA), and $1540 \mathrm{~cm}^{-1}$ (DNA/RNA), and $1585 \mathrm{~cm}^{-1}$ (DNA/RNA, in-plane ring vibration). ${ }^{15-18}$

In a separate experiment, SERS spectra induced by incubated gold nanoparticles (10 and $50 \mathrm{~nm}$ ) were collected and compared with the IGAuN-induced SERS (Fig. 4). No SERS spectra could be collected from the controls (cells without AuNPs or cells without IGAuNs). A striking difference between the IGAuN- and AuNP-induced SERS spectra is the sharp Raman band around $500 \mathrm{~cm}^{-1}$, which was absent in the IGAuN-induced SERS. This band is also present in almost all the reported AuNP SERS spectra from previous studies. ${ }^{3,19}$ This can be related to the stretching vibration mode of disulfide bond $(\nu \mathrm{S}-\mathrm{S})$. This can be indicative of lysosomal proteins that have an abundance of disulfide bonds. Lysosomal proteins need to resist the hostile conditions of the environment in which they reside. The oxidizing environment of lysosomes as opposed to the reducing environment of the cytosol favors the formation of disulfide bond. ${ }^{20-22}$ This further evidence indicates that IGAuNs in contrast to AuNPs is not translocated to the endosomes or lysosomes and hence could potentially serve as a SERS probe for sensing the cytoplasmic/nuclear environment. TEM imaging supports this observation [Fig. 1(g)]. Prevalence of protein fingerprints in both the IGAuN- and AuNP-induced SERS spectra could be related to the fact that proteins have an affinity to bind to the surface of gold nanoparticles through chemisroption.

In summary, IGAuN is introduced for the first time as a potential SERS substrate to probe the cytoplasm and nucleus of single cells. This capability can prove to be a promising SERS platform to study biochemical mechanisms or interactions, and in elucidating the enzymatic/molecular mechanism involved in the bioreduction of gold itself. Future studies will focus on optimizing this platform by testing different human cell lines, and controlling the shape and distribution of IGAuNs and refining the experimental designs for higher sensitivity and selectivity. Controlling the growth of gold nanoparticles inside the cell is a critical step toward generating SERS-favorable IGAuNs and obtaining more meaningful information.

\section{Acknowledgments}

We thank Molecular Cytometry Lab and Electron Microscopy Facility personnel of Purdue University for their help.Mr. Patrick Dover from Olympus Incorporated is acknowledged for assistance in providing water immersion objectives. This research was conducted at the Physiological Sensing Facility.

\section{References}

1. I. Chourpa, H. Morjani, J. F. Riou, and M. Manfait, "Intracellular molecular interactions of antitumor drug amsacrine (m-AMSA) as revealed by surface-enhanced Raman spectroscopy," FEBS Lett. 397, 61-64 (1996).

2. C. E. Talley, L. Jusinski, C. W. Hollars, S. M. Lane, and T. Huser, "Intracellular $\mathrm{pH}$ sensors based on surface-enhanced Raman scattering," Anal. Chem. 76, 7064-7068 (2004).

3. K. Kneipp, A. S. Haka, H. Kneipp, K. Badizadegan, N. Yoshizawa, C. Boone, K. E. Shafer-Peltier, J. T. Motz, R. R. Dasari, and M. S Feld, "Surface-enhanced Raman Spectroscopy in single living cells using gold nanoparticles," Appl. Spectrosc. 56, 150-154 (2002).

4. J. Kneipp, H. Kneipp, W. L. Rice, and K. Kneipp, "Optical probes for biological applications based on surface-enhanced Raman scattering from indocyanine green on gold nanoparticles," Anal. Chem. 77, 2381-2385 (2005).

5. B. D. Chithrani, A. A. Ghazani, and W. C. W. Chan, "Determining the size and shape dependence of gold nanoparticle uptake into mammalian cells," Nano Lett. 6, 662-668 (2006)

6. K. Kneipp, H. Kneipp, and J. Kneipp, "Surface-enhanced Raman scattering in local optical fields of silver and gold nanoaggregatessfrom single-molecule Raman spectroscopy to ultrasensitive probing in live cells," Acc. Chem. Res. 39, 443-450 (2006).

7. A. Wei, B. Kim, B. Sadtler, and S. L. Tripp, "Tunable surfaceenhanced Raman scattering from large gold nanoparticle arrays," ChemPhysChem 2, 743-+ (2001).

8. D. Mandal, M. E. Bolander, D. Mukhopadhyay, G. Sarkar, and P. Mukherjee, "The use of microorganisms for the formation of metal nanoparticles and their application," Applied Microbio. Biotechnol. 69, 485-492 (2006).

9. J. R. Lloyd, "Microbial reduction of metals and radionuclides," FEMS Microbiol. Rev. 50, 411-425 (2003).

10. A. Anshup, J. S. Venkataraman, C. Subramaniam, R. R. Kumar, S. Priya, T. R. S. Kumar, R. V. Omkumar, A. John, and T. Pradeep, "Growth of gold nanoparticles in human cells," Langmuir 21, 11562-11567 (2005)

11. J. F. Hainfeld and R. D. Powell, "New frontiers in gold labelling," J. Histochem. Cytochem. 48, 471-480 (2000).

12. G. Danscher and J. O. R. Norgaard, "Light microscopic visualization of colloidal gold on resin-embedded tissue," J. Histochem. Cytochem. 31, 1394-1398 (1983)

13. D. V. Goia and E. Matijevic, "Preparation of monodispersed metal particles," New J. Chem. 22, 1203-1215 (1998).

14. S. Senapati, D. Mandal, A. Ahmad, M. I. Khan, M. Sastry, and R. Kumar, "Fungus mediated synthesis of silver nanoparticles: a novel biological approach," Indian J. Phys. Proc. Indian Assoc. Cultivation Sci. Part A 78A, 101-105 (2004).

15. C. Krafft, T. Knetschke, R. H. W. Funk, and R. Salzer, "Identification of organelles and vesicles in single cells by Raman microspectroscopic mapping," Vib. Spectrosc. 38, 85-93 (2005).

16. D. Naumann, "FT-infrared and FT-Raman spectroscopy in biomedical research," Appl. Spectrosc. Rev. 36, 239-298 (2001).

17. K. M. Omberg, J. C. Osborn, S. L. L. Zhang, J. P. Freyer, J. R. Mourant, and J. R. Schoonover, "Raman spectroscopy and factor analysis of tumorigenic and non-tumorigenic cells," Appl. Spectrosc. 56, 813-819 (2002).

18. F. R. Dollish, W. G. Fateley, and F. F. Bentley, Characteristic Raman Frequencies of Organic Compounds, Wiley, New York (1974).

19. C. Eliasson, A. Loren, J. Engelbrektsson, M. Josefson, J. Abrahamsson, and K. Abrahamsson, "Surface-enhanced Raman scattering imaging of single living lymphocytes with multivariate evaluation," Spectrochim. Acta, Part A 61, 755-760 (2005).

20. J. Hu, R. S. Sheng, Z. S. Xu, and Y. E. Zeng, "Surface-enhanced Raman-spectroscopy of lysozyme," Spectrochim. Acta, Part A 51, 1087-1096 (1995).

21. U. T. Phan, R. L. Lackman, and P. Cresswell, "Role of the C-terminal propeptide in the activity and maturation of gamma-interferoninducible lysosomal thiol reductase (GILT)," Proc. Natl. Acad. Sci. U.S.A. 99, 12298-12303 (2002).

22. H. Ostergaard, C. Tachibana, and J. R. Winther, "Monitoring disulfide bond formation in the eukaryotic cytosol," J. Cell Biol. 166, 337-345 (2004). 